\title{
Predictors of ventricular remodelling in patients with reperfused acute myocardial infarction and left ventricular dysfunction candidates for bone marrow cell therapy: insights from the BONAMI trial
}

\author{
Alain Manrique ${ }^{1,2,17}$ - Patricia Lemarchand ${ }^{3,4,5,6}$ - Béatrice Delasalle ${ }^{3,4,5,6}$. \\ Olivier Lairez $^{7}$ - Catherine Sportouch-Duckan ${ }^{8,9}$ - Guillaume Lamirault G,4,5,6 $_{\text {. }}$ \\ Philippe Le Corvoisier $^{10}$ • Yannick Neuder ${ }^{11}$ - Marjorie Richardson ${ }^{12}$ - Alain Lebon ${ }^{13}$. \\ Jérome Roncalli $^{7}$ • Christophe Piot ${ }^{8,9}$ - Jean-Noel Trochu ${ }^{3,4,5,6}$ - Emmanuel Teiger ${ }^{14}$. \\ Claude Hossein-Foucher ${ }^{15,16}$ • Thierry Le Tourneau ${ }^{3,4,5,6}$
}

Received: 31 July 2015 / Accepted: 1 December 2015

(C) Springer-Verlag Berlin Heidelberg 2015

\begin{abstract}
Purpose Few data are available regarding the relation of left ventricular (LV) mechanical dyssynchrony to remodelling after acute myocardial infarction (MI) and stem cell therapy. We evaluated the 1-year time course of both LV mechanical dyssynchrony and remodelling in patients enrolled in the BONAMI trial, a randomized, multicenter controlled trial assessing cell therapy in patients with reperfused MI.
\end{abstract}

Electronic supplementary material The online version of this article (doi:10.1007/s00259-015-3279-z) contains supplementary material, which is available to authorized users.

Alain Manrique

manrique@cyceron.fr

Nuclear Medicine, CHU de Caen, Caen F-14000, France

2 EA 4650, Université de Caen Normandie, Caen F-14000, France

3 INSERM, UMR1087, l'institut du thorax, Nantes F-44000, France

4 CNRS, UMR 6291, Nantes F-44000, France

5 Université de Nantes, Nantes F-44000, France

6 CHU de Nantes, Nantes F-44000, France

7 Cardiac Imaging Center, CIC Biothérapies, CHU de Toulouse, Institut CARDIOMET-Toulouse, Toulouse, France

8 Institut de Génomique Fonctionnelle, INSERM U661, CNRS UMR 5203, Université Montpellier, Montpellier, France

9 Clinique du Millénaire, Montpellier, France
Methods Patients with acute MI and ejection fraction $(\mathrm{EF}) \leq 45 \%$ were randomized to cell therapy or to control and underwent thallium single-photon emission computed tomography (SPECT), radionuclide angiography, and echocardiography at baseline, 3 months, and 1 year. Eighty-three patients with a comprehensive 1-year follow-up were included. LV dyssynchrony was assessed by the standard deviation (SD) of the LV phase histogram using radionuclide angiography.

10 INSERM, Centre d'Investigation Clinique 1430 et U955 équipe 3, Hôpital Henri Mondor, Créteil, France

11 Pole Thorax et Vaisseaux, CHU de Grenoble, Grenoble, France

12 Service d'Explorations Fonctionnelles Cardiovasculaires, Hôpital Cardiologique, CHRU Lille, Lille, France

13 Service de Cardiologie, CHU de Caen, Caen F-14000, France

14 AP-HP, unité de cardiologie interventionnelle et fédération de cardiologie, Hôpital Henri Mondor, Créteil, France

15 Service de médecine nucléaire, Hôpital Salengro CHRU de Lille, Lille, France

16 UFR de Médecine, Université de Lille 2, Lille, France

17 CHU de Caen et GIP Cyceron, Campus Jules Horowitz, BP 5229, 14074 Caen cedex 6, France 
Remodelling was defined as a $20 \%$ increase in LV endsystolic volume index (LVESVI) at 1 year.

Results At baseline, LVEF, wall motion score index, and perfusion defect size were significantly impaired in the 43 patients (52\%) with LV remodelling (all $p<0.001$ ), without significant increase in LV mechanical dyssynchrony. During follow-up, there was a progressive increase in LV SD $(p=0.01)$. Baseline independent predictors of LV remodelling were perfusion SPECT defect size $(p=0.001)$, LVEF $(p=0.01)$ and a history of hypertension $(p=0.043)$. Bone marrow cell therapy did not affect the time-course of LV remodelling and dyssynchrony.

Conclusions LV remodelling 1 year after reperfused MI is associated with progressive LV dyssynchrony and is related to baseline infarct size and ejection fraction, without impact of cell therapy on this process.

Keywords Myocardial infarction · Ventricular remodelling · Dyssynchrony · Single photon emission computer tomography $\cdot$ Echocardiography $\cdot$ Cell therapy

\section{Introduction}

The improvements in treatment of acute myocardial infarction (MI), aimed to restore persistent myocardial perfusion, preserve myocyte integrity, and prevent left ventricular (LV) dysfunction, have resulted in a better early survival. However, with more patients surviving the initial stage of AMI, postinfarction LV remodelling occurs in a relevant proportion of patients [1]. Although the remodelling process tends to restore stroke volume despite a depressed ejection fraction, ventricular dilation is associated with a poor long-term clinical outcome [2]. Infarct size, infarct-related artery patency, anterior location, LV end-systolic volume, and microvascular obstruction are clinical predictors of LV remodelling. Recent results suggested that early LV dyssynchrony has an additional detrimental impact on LV function [3] and could predict the occurrence of $\mathrm{LV}$ remodelling $[4,5]$. However, it remains unclear whether LV dyssynchrony observed soon after acute MI is independently associated with LV remodelling.

In the setting of irreversible myocyte loss following an acute myocardial infarction, and given the poor endogenous regenerative capacity of the heart, stem cell therapy has emerged as a unique opportunity for cardiac repair. However, a meta-analysis of randomized control trials demonstrated a poor long term improvement of left ventricular function after stem cell therapy, showing only a $3 \%$ increase in left ventricular ejection fraction, which remains the most frequently assessed surrogate endpoint [6].

Accordingly, this study was conducted to assess the determinants of ventricular remodelling in patients with reperfused myocardial infarction and left ventricular dysfunction eligible for stem cell therapy. To achieve this goal, we assessed the 1year time course of ventricular remodelling in a cohort of patients prospectively included in the BONAMI trial, a study dedicated to evaluate the effect of bone marrow-derived cell therapy on myocardial viability [7].

\section{Material and methods}

Patient population The BONAMI study was a randomized, multicentre controlled trial assessing cell therapy in patients with acute MI. Screening criteria were: age 18-75 years, a successful percutaneous coronary intervention (PCI) with bare metal stent implantation performed on the culprit lesion during the $24 \mathrm{~h}$ after the onset of symptoms, and LVEF $<50 \%$ assessed by echocardiography. The culprit artery was defined as an occluded artery or by a severe stenosis secondary to superimposed thrombus. Randomization criteria were defined as follows: $\mathrm{LVEF} \leq 45 \%$ assessed by RNA and absence of myocardial viability in at least $2 / 17$ contiguous segments by SPECT. Main exclusion criteria were: age $>75$ years; previous myocardial infarction; patient instability after myocardial infarction; cardiac disease except ischemic heart disease; multivessel coronary artery disease; need for coronary revascularization in the future; pulmonary edema and cardiogenic shock; advanced renal or hepatic failure; non-cardiac disease adversely affecting prognosis; coagulopathy, thrombocytopenia, and leukopenia. As previously described [7], 101 patients met the inclusion criteria and were randomized to cell therapy $(n=52)$ or to control $(n=49)$. After inclusion, patients underwent thallium perfusion SPECT, equilibrium radionuclide angiography and echocardiography at baseline, 3 months, and 1 year follow-up. In the present study, the population consisted of 83 patients with a comprehensive follow-up at 1 year. The ethics review board of Nantes University Hospital approved the protocol, and the study was conducted in accordance with the Declaration of Helsinki. All subjects gave informed consent.

Radionuclide equilibrium angiography Planar equilibrium radionuclide angiography (RNA) was performed in all patients and left (LVEF), and right ejection fraction (RVEF) was calculated using a count-based method [8]. The mean phase angle and phase standard deviation were derived from the phase histograms. Left ventricular mechanical synchrony was assessed as the standard deviation of the left ventricular phase histogram (LV SD). Interventricular contractile synchrony was calculated as the absolute difference between LV and RV mean phase angles (RV-LV). Results were expressed in milliseconds (ms).

Myocardial perfusion imaging Myocardial perfusion SPECT was acquired $15 \mathrm{~min}$ and 3-4 h after a rest injection 
of thallium-201 (1.5 MBq/kg). SPECT data were analyzed using a 17-segment division of the left ventricle [9] by consensus of two experienced observers blinded from any clinical data. Infarct extent was calculated using an automated software and expressed as the percentage of LV showing a thallium uptake $<60 \%$. This latter criterion was applied on early images at the acute phase, and on redistribution images at follow-up, according to previous results showing that within the first week after myocardial infarction, early thallium imaging better reflects myocardial viability and functional recovery [10].

Echocardiography All echocardiography examinations were recorded and analyzed off-line by experienced investigators blinded from any clinical data. Left ventricular end-diastolic and end-systolic diameter (LVEDD and LVESD) were measured in the parasternal long axis view. Left ventricular volumes (biplane modified Simpson rule) and ejection fraction were measured from four-chamber apical views and volumes were indexed to body surface area (LVEDVI and LVESVI) [11]. Wall motion score index (WMSI) was calculated using a 17-segment model [9] and a 4-point grading system from 1 (normal) to 4 (dyskinetic). Pulmonary artery systolic pressure (PASP) was determined using the modified Bernouilli equation. Tissue Doppler profiles were recorded at the medial and lateral mitral annulus from the apical four-chamber view. Systolic tissue velocity (S), early diastolic (E'), and late diastolic (A') tissue velocities were recorded, and the septal and lateral E/E' ratio were calculated.

Statistical analysis Results were expressed as mean $\pm \mathrm{SD}$ or number (percentages) as appropriate. Comparisons between groups were performed with Student's $t$ tests or $\chi^{2}$ tests. Correlations were presented with Pearson coefficient ( $r, p$-value). Adjusted correlations between variables were assessed by linear regression analysis. Logistic regression analysis was used to analyze the determinants of LV mechanical dyssynchrony or remodelling and LVEF impairment (odds ratio with $95 \%$ CI). These parameters were: clinical characteristics, MI treatment, radionuclide, and echocardiographic parameters. To analyze repeated measures over time (imaging parameters), we used mixed models and adjusted Tukey tests. All models were adjusted by the treatment allocated (control or bone marrow cell therapy) and interactions were tested (only $p$-values $<0.05$ were maintained).

Left ventricular remodelling is a complex process and involves different pathophysiological mechanisms over time [12]. To account for this phenomenon, the predictive value of potential predictors of late remodelling was analyzed both at baseline and 3 months after myocardial infarction. The presence of $\mathrm{LV}$ remodelling was defined as a relative increase of at least $20 \%$ in LVESVI between baseline and 1-year follow-up. In addition, indexed end-systolic volumes were analyzed as a continuous variable and predictors of ESVI at 1-year follow-up were determined. All analyses were performed using SAS 9.1 statistical software (SAS Institute, Cary, NC, USA). A $p$-value $<0.05$ was considered statistically significant.

\section{Results}

Baseline data Eighty-three patients with a comprehensive follow-up at 1 year were included. During primary PCI, TIMI flow grade III was obtained in 78 patients $(99 \%)$. The patients included in the study had large myocardial infarctions, as demonstrated by high peak troponin levels and a large perfusion SPECT defect resulting in left ventricular dilation and systolic dysfunction (see Table 1). Medical therapy was not different between patients groups (see supplemental data). The delay between myocardial infarction and baseline investigations was as follows: planar RNA, $4 \pm 2$ days; thallium SPECT, $5 \pm 3$ days; echocardiography, $7 \pm 2$ days.

LV remodelling at 1-year follow-up A preliminary statistical analysis of 3 months and 1 year functional results showed no difference between both patients groups (control group and group receiving cardiac cell therapy) for LVESVI and LVEF. Therefore, all patients were further analysed together, independently of cardiac cell therapy. During the first year after MI, there was a progressive impairment of global left ventricular function, with a significant increase in both end-diastolic $(p<0.0001)$ and end-systolic $(p<0.0001)$ volume indexes (see Table 2). Along with LV dilation, both inter- and intraventricular dyssynchrony progressively increased during follow-up, particularly in patients with LV remodelling (see Fig. 1). Similarly, there was also a progressive left atrial remodelling process. The treatment regimen allocated (control or bone marrow cell therapy) did not modify the time-course of LV remodelling and dyssynchrony.

Using univariate linear regression, the baseline parameters associated with ESVI measured at 1-year follow-up were: LV SD ( $p=0.03)$, LVEF, LVESVI, LVEDVI, WMSI, and thallium SPECT defect size (all $p$ values $<0.0001$ ). Among the parameters measured 3 months after MI, those associated with ESVI measured at 1-year follow-up included LVESVI, LVEDVI, LVEF, WMSI, LVEDDI, LVESDI, thallium SPECT defect size, and LV SD (all $p$ values $<0.0001$ ). Using multivariate analysis, the independent predictors of ESVI at 1year follow-up were, at baseline: LVEF $(p<0.0001)$ and thallium SPECT defect size $(p=0.002)$, and at 3 months: ESVI $(p<0.0001)$ and WMSI $(p=0.035)$.

The study population was divided into two groups of patients with $(n=43,52 \%)$ and without LV remodelling $(n=40$, $48 \%$ ). At baseline (see Table 1), patients with LV remodelling at 1 year showed a larger infarct size (documented by 
Table 1 Baseline characteristics of patients without vs. with left ventricular remodelling

\begin{tabular}{|c|c|c|c|c|}
\hline & $\begin{array}{l}\text { All subjects } \\
(n=83)\end{array}$ & $\begin{array}{l}\text { No remodelling } \\
(n=40)\end{array}$ & $\begin{array}{l}\text { remodelling } \\
(n=43)\end{array}$ & $p$-value \\
\hline \multicolumn{5}{|l|}{ Clinical characteristics at baseline } \\
\hline Age (years; mean $\pm \mathrm{SD}$ ) & $55.3 \pm 11.3$ & $55.1 \pm 12.3$ & $55.6 \pm 10.5$ & NS \\
\hline BMC group \% (n) & $54(45)$ & $45(18)$ & $63(27)$ & NS \\
\hline Male gender \% (n) & $88(73)$ & $95(38)$ & $81(35)$ & NS \\
\hline Hypertension \% (n) & $37(31)$ & $25(10)$ & $49(21)$ & 0.030 \\
\hline Hyperlipidemia \% (n) & $43(36)$ & $38(15)$ & $49(21)$ & NS \\
\hline Diabetes \% (n) & $22(18)$ & $23(9)$ & $21(9)$ & NS \\
\hline Current smoker \% (n) & $54(45)$ & $53(21)$ & $56(24)$ & NS \\
\hline QRS duration (ms) & $89.8 \pm 17.1$ & $90.0 \pm 17.7$ & $89.6 \pm 16.7$ & NS \\
\hline Peak troponin level $(\mu \mathrm{g} / \mathrm{L})$ & $212.6 \pm 227.5$ & $157.3 \pm 152.1$ & $260.0 \pm 269.2$ & 0.039 \\
\hline $\begin{array}{l}\text { Time to revascularization } \\
(<12 \mathrm{~h}) \%(\mathrm{n})\end{array}$ & $73(61)$ & $73(29)$ & $74(32)$ & NS \\
\hline $\begin{array}{l}\text { Infarct-related artery } \\
\text { (LAD) \% (n) }\end{array}$ & $95(79)$ & $90(36)$ & $100(43)$ & 0.050 \\
\hline TIMI flow 2-3 after PCI \% (n) & $99(78)$ & $100(38)$ & $98(40)$ & NS \\
\hline \multicolumn{5}{|l|}{ Radionuclide parameters } \\
\hline $\begin{array}{l}\text { Perfusion SPECT Defect } \\
\text { size \% (n) }\end{array}$ & $31.1 \pm 16.3$ & $24.6 \pm 15.0$ & $37.1 \pm 15.2$ & $<0.0001$ \\
\hline RNA LVEF (\%) & $37.3 \pm 8.1$ & $40.0 \pm 7.6$ & $34.9 \pm 7.9$ & 0.005 \\
\hline RNA RVEF (\%) & $46.6 \pm 10.2$ & $45.8 \pm 9.5$ & $47.2 \pm 10.9$ & NS \\
\hline LV SD (ms) & $75.6 \pm 24.4$ & $72.1 \pm 24.9$ & $78.7 \pm 23.8$ & NS \\
\hline LV-RV delay (ms) & $24.7 \pm 52.8$ & $15.7 \pm 50.6$ & $32.8 \pm 54.0$ & NS \\
\hline \multicolumn{5}{|l|}{ Echocardiographic parameters } \\
\hline LVEDVI $\left(\mathrm{mL} / \mathrm{m}^{2}\right)$ & $57.9 \pm 14.9$ & $59.1 \pm 14.4$ & $56.7 \pm 15.3$ & NS \\
\hline $\operatorname{LVESVI}\left(\mathrm{mL} / \mathrm{m}^{2}\right)$ & $35.8 \pm 12.1$ & $35.1 \pm 10.6$ & $36.5 \pm 13.4$ & NS \\
\hline LVEF $(\%)$ & $38.9 \pm 7.6$ & $41.3 \pm 6.5$ & $36.8 \pm 8.0$ & 0.006 \\
\hline WMSI & $2.4 \pm 0.3$ & $2.3 \pm 0.3$ & $2.5 \pm 0.3$ & 0.001 \\
\hline E/E' septal ratio & $12.1 \pm 4.5$ & $12.1 \pm 4.4$ & $12.1 \pm 4.6$ & NS \\
\hline $\mathrm{E} / \mathrm{E}^{\prime}$ lateral ratio & $10.3 \pm 5.6$ & $10.7 \pm 4.5$ & $9.9 \pm 6.4$ & NS \\
\hline Systolic PAP (mmHg) & $31.5 \pm 10$ & $31.2 \pm 9.4$ & $31.7 \pm 10.8$ & NS \\
\hline
\end{tabular}

$B P$ indicates blood pressure, $B M C$ bone marrow cell, $C A D$ coronary artery disease, $L A D$ left anterior descending coronary artery, $P C I$ percutaneous coronary intervention, $M I$ myocardial infarction, $A C E$ angiotensin-converting enzyme, $R N A$ radionuclide angiography, $L V E F$ left ventricular ejection fraction, $R V E F$ right ventricular ejection fraction, $L V$ left ventricle, $R V$ right ventricle, $S D$ standard deviation of the phase histogram, $L V E D V I$ left ventricular end-diastolic volume index, $L V E S V I$ left ventricular end-systolic volume index, WMSI wall motion score index, $P A P$ pulmonary artery pressure increased peak troponin level and perfusion SPECT defect size), and an impairment of LV function demonstrated by decreased WMSI and LVEF compared to patients without LV remodelling. In addition, they were more likely to have a culprit lesion on the left anterior descending coronary artery and a history of hypertension. At 1 year follow-up, LV SD correlated to RNA LVEF ( $r=-0.64 . p<0.0001)$, LVESVI $(r=0.54, p<0.0001)$, LVEDVI $(r=0.55, p<0.0001)$, perfusion SPECT defect $(r=0.33, p=0.003)$ and peak troponin $(r=0.24, p=0.037)$. No significant differences in LV mechanical dyssynchrony parameters were observed between the two groups of patients.

Univariate predictors of LV remodelling at 1-year followup are depicted in Table 3. Using logistic multivariate analysis
$(N=79)$, independent predictors at baseline were RNA LVEF (OR: $0.138,95 \%$ confidence interval: $0.053-6.686, p=0.01$ ), perfusion SPECT defect size (OR: $-0.073,95 \%$ confidence interval: $0.022-11.390, p=0.001)$ and hypertension (OR: $0.607,95 \%$ confidence interval: $0.300-4.094, p=0.04$ ). Among the parameters assessed at 3-month follow-up, LVEDD (OR: 1.133, 95 \% confidence interval: 1.017-1.23, $p=0.02$ ) was an independent predictor of remodelling.

\section{Discussion}

Despite encouraging initial results, cell therapy demonstrated a significant, but modest improvement of left ventricular 
Table 2 Time course of imaging parameters

\begin{tabular}{lllll}
\hline & Baseline & 3 months & 1 year & Global $p$-value \\
\hline RNA LVEF (\%) & $37 \pm 8.5^{\S / * *}$ & $40 \pm 10.3$ & $39 \pm 10.7$ & 0.0014 \\
RNA RVEF (\%) & $46.3 \pm 10.1^{* *}$ & $48.5 \pm 9.1$ & $48.7 \pm 8.9$ & 0.0375 \\
Perfusion Defect size (\%) & $30.6 \pm 16^{\ddagger / \S \S}$ & $23.8 \pm 13$ & $26.6 \pm 16.6$ & $<0.0001$ \\
LV SD (ms) & $75.1 \pm 24^{* *}$ & $76.8 \pm 36.7$ & $85.5 \pm 37.9$ & 0.0101 \\
LV-RV delay (ms) & $24.6 \pm 53.8^{\ddagger / \S \S}$ & $14.2 \pm 42.3^{\dagger \ddagger}$ & $33.5 \pm 45$ & 0.0122 \\
LVEDVI & $57.5 \pm 14.4^{\ddagger / 4 \ddagger}$ & $68.1 \pm 21.4$ & $71.6 \pm 25.5$ & $<0.0001$ \\
LVESVI & $35.7 \pm 11.7^{\ddagger / * \ddagger}$ & $42 \pm 19$ & $44.7 \pm 21.7$ & $<0.0001$ \\
LVEDD & $27 \pm 3.4^{\dagger / * \hbar}$ & $28.3 \pm 3.2$ & $28.9 \pm 3.6$ & $<0.0001$ \\
LVESD & $18.9 \pm 3.6^{\dagger / * \star}$ & $20.6 \pm 3.5$ & $21.4 \pm 4.1$ & $<0.0001$ \\
WMSI & $2.4 \pm 0.3^{\S}$ & $2.3 \pm 0.4$ & $2.3 \pm 0.4$ & 0.002 \\
Left atrial diastolic diameter & $19.7 \pm 2.8^{\dagger / * *}$ & $20.8 \pm 2.7$ & $20.5 \pm 2.7$ & 0.0007 \\
Left atrial volume index & $28.8 \pm 10^{*}$ & $33.8 \pm 11.3^{\S \S}$ & $41.1 \pm 22$ & $<0.0001$ \\
E/E' septal ratio & $11.9 \pm 4.4$ & $12.1 \pm 5.4$ & $13.1 \pm 5.9$ & NS \\
E/E' lateral ratio & $10.1 \pm 5.3$ & $9.3 \pm 4.2$ & $10 \pm 6.2$ & NS \\
Systolic PAP (mmHg) & $31.5 \pm 9.8$ & $32.4 \pm 9.7$ & $33.8 \pm 9.7$ & NS
\end{tabular}

$R N A$ indicates radionuclide angiography, $L V E F$ left ventricular ejection fraction, $R V E F$ right ventricular ejection fraction, $S D$ standard deviation of the phase histogram, $L V E D V I$ left ventricular end-diastolic volume index, LVESVI left ventricular end-systolic volume index, $L V E D D$ left ventricular end-diastolic diameter, $L V E S D$ left ventricular end-systolic diameter, WMSI wall motion score index, $P A P$ pulmonary artery pressure

${ }^{*} p<0.05,{ }^{\S} p<0.01,{ }^{\dagger} p<0.0,01$ and ${ }^{\star} p<0.0001$ vs. 3 months

${ }^{* *} p<0.05,{ }^{\S \S} p<0.01$, and ${ }^{\sharp t} p<0.0001$ vs. 1 year function in humans. There are a lack of data analyzing the determinants of left ventricular remodelling in patients receiving cell therapy that may help to enhance the efficacy of regenerative strategies. The relationship between early LV asynchrony observed after an acute MI and late LV remodelling is still controversial and has not been investigated in this population. The main findings of this study can be summarized as follows: 1) One half of the patients included in the BONAMI trial exhibited significant remodelling at 1 year after acute myocardial infarction despite an optimal management; 2) at baseline, the independent predictors of remodelling included
LVEF, perfusion SPECT defect and a history of hypertension; 3) dyssynchrony at baseline was not predictive of remodelling in this population; and finally 4) ventricular remodelling was not significantly impacted by bone marrow cell therapy.

\section{Incidence of left ventricular remodelling Although LV} function is commonly used as a surrogate endpoint for efficacy of cell therapy, the incidence of ventricular remodelling at 1 year after MI remains unknown in patients prospectively enrolled in clinical trials [6, 13-16]. During follow-up, $52 \%$ of our study population demonstrated a significant
Fig. 1 Time course of left ventricular dyssynchrony according to left ventricular remodelling, at baseline, $\mathrm{M} 3$, and M12. ${ }^{*} p \leq 0.05$

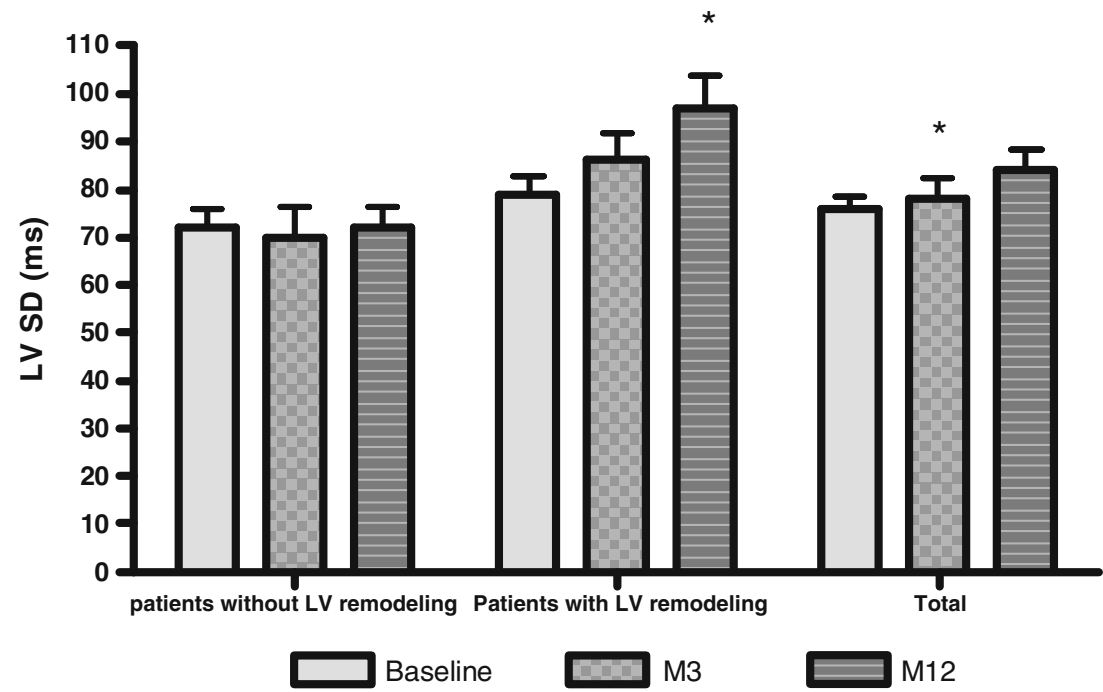


Table 3 Univariate logistic regression analysis of baseline and 3 months predictors of left ventricular remodelling at 1 -year follow-up

\begin{tabular}{llll}
\hline & OR & $95 \%$ CI & $p$-value \\
\hline Baseline parameters & & & \\
RNA LVEF & 0.92 & $0.86-0.98$ & 0.009 \\
Echo LVEF & 0.92 & $0.86-0.98$ & 0.009 \\
WMSI & 16.62 & $2.84-97.28$ & 0.002 \\
LVEDVI & 0.99 & $0.96-1.02$ & NS \\
LVESVI & 1.01 & $0.97-1.05$ & NS \\
Perfusion SPECT defect size & 1.06 & $1.02-1.09$ & 0.001 \\
Peak troponin level & 1.00 & $1.00-1.01$ & 0.05 \\
Cell therapy (control vs. BMC) & 0.49 & $0.20-1.17$ & $\mathrm{NS}$ \\
HTA (no vs. yes) & 2.86 & $1.13-7.27$ & 0.03 \\
Non critical stenoses on non culprit arteries & 0.798 & $0.259-2.458$ & $\mathrm{NS}$ \\
3 months parameters & & & 0.001 \\
RNA LVEF & 0.91 & $0.86-0.96$ & 0.001 \\
Echo LVEF & 0.91 & $0.86-0.96$ & $<0.0001$ \\
WMSI & 21.87 & $4.67-102.4$ & 0.03 \\
LVEDVI & 1.03 & $1.00-1.05$ & 0.007 \\
LVESVI & 1.05 & $1.01-1.08$ & 0.003 \\
Perfusion SPECT defect size & 1.06 & $1.02-1.11$ & 0.02 \\
LVEDD & 1.10 & $1.02-1.20$ & $\mathrm{NS}$ \\
LVESD & 1.07 & $0.99-1.14$ & \\
\hline
\end{tabular}

$R N A$ indicates radionuclide angiography, $L V E F$ left ventricular ejection fraction, WMSI wall motion score index, LVESVI left ventricular end-systolic volume index, $L V E D V I$ left ventricular end-diastolic volume index, SPECT single photon emission computed tomography, LVEDD left ventricular end-diastolic diameter, LVESV left ventricular end-systolic diameter remodelling of the left ventricle, which is higher than previously observed after acute myocardial infarction. In a recent study, Mollema et al. [17] reported a significant remodelling (defined as an absolute increase in LVESV $\geq 15 \%$ ) in $20 \%$ of patients. Using the end-diastolic volume index as a marker of remodelling, Giannuzzi et al. [18] found severe remodelling in $16 \%$ of patients. These latter studies investigated non-selected patients with a relatively preserved LV function (mean LVEF from 46 to $48 \%$ ) compared to our study. In patients with larger MI, Bolognese et al. [1] reported LV remodelling in a higher proportion $(30 \%)$ of patients. The high incidence of ventricular remodelling in our study is likely due to the enrolment of selected patients with LV dysfunction and large myocardial infarcts.

\section{Dyssynchrony and predictors of left ventricular remodel-} ling Cardiac remodelling after MI is associated with poor event-free survival [1]. White et al. [19] early showed that end-systolic volume was the most powerful predictor of death after myocardial infarction. On the other hand, left ventricular recovery after successful primary PCI is associated with a better long-term prognosis [2]. Primary PCI and pharmacologic therapies targeting the rennin-angiotensin system and neuro-hormonal activation have shown a beneficial impact on remodelling [20-23]. However, the early identification of patients at high risk of remodelling after MI is of paramount importance.

Recent data suggested a relationship between remodelling and dyssynchrony. Using 2D speckle tracking, Mollema et al. [17] evaluated 194 consecutive patients admitted with an acute myocardial infarction treated by primary PCI. In this study, dyssynchrony showed the strongest correlation with the incidence of remodelling at 6 months of follow-up, even after adjustment for the peak level of troponin, hypertension, baseline LVESV, and baseline LVEDV. In a series of 47 patients, Zhang et al. [4] demonstrated that patients with remodelling (defined in this study as an increase in LVESV $\geq 10 \%$ at 1 year) had a higher extent of early dyssynchrony and infarct size assessed by contrast-enhanced cardiac magnetic resonance. However, they also found that dyssynchrony was correlated with WMSI, ESV, EDV, LVEF, and infarct size at baseline. Nucifora et al. [3] further demonstrated that patients with mechanically reperfused infarction and LVEF $\leq 45 \%$ had a higher extent of dyssynchrony and increased infarct size using contrast echocardiography. Finally, Turan et al. [24] found a sensitivity of $73 \%$ of dyssynchrony for predicting remodelling after a first MI treated successfully by PCI.

In the present study, we found no difference in terms of dyssynchrony at baseline between patients with and without remodelling. The BONAMI trial enrolled patients with large 
infarcts (mean infarct size $=31 \%$ of the LV) and LV dysfunction (mean LVEF: $39 \%$ ), two factors that are correlated to ventricular dyssynchrony [25]. As a consequence, even patients without LV remodelling at follow-up had dyssynchrony at baseline. Moreover, the culprit artery was the LAD in all but four patients in our study population. Previous findings demonstrated that in patients with anterior infarction, dyssynchrony is increased compared to patients with inferior infarction, even in patients with normal QRS duration [25, 26]. Thus, assessing patients with anterior infarction might also contribute to the low predictive value of phase analysis, as anterior segments have a higher contribution to observed dispersion with respect to inferior and posterior segments. Nucifora et al. [27] reported temporal evolution of LV dyssynchrony after reperfused myocardial infarction and observed that dyssynchrony changes were associated with changes in LVEF. In the present study, although there was a progressive increase of dyssynchrony over time, baseline inter- or intra-ventricular mechanical delays was not predictive of ventricular remodelling. The use of the planar technique, that analyses dyssynchrony on a projection of the overall cardiac volume, may have contributed to the low predictive value of phase analysis in this study. It is likely that planar imaging does not accurately discriminate the respective contributions of the infarct zone and the remote territory. The assessment of left ventricular mechanical contraction as determined by phase analysis of ECG-gated myocardial perfusion SPECT has been recently proposed to overcome this issue [28]. Henneman et al. [29] demonstrated that the response to cardiac resynchronization therapy is related to the presence of LV dyssynchrony assessed by phase analysis from gated myocardial perfusion SPECT, including in patients with ischemic cardiomyopathy. These results suggest that the usage of SPECT techniques could be more appropriate for the assessment of left ventricular dyssynchrony in patients with large myocardial scars.

Infarct size and viability are strong predictors of clinical outcome after myocardial infarction. The prognostic value of infarct size has been early established [30] using myocardial perfusion SPECT. The extent of infarct-zone viability was reported as inversely correlated with further changes in end-diastolic volumes at 6 months [31]. Moreover, perfusion SPECT performed within 1 month after the acute phase of infarction could predict ventricular enlargement with a high accuracy [32, 33]. Our results demonstrated that the independent predictors of a significant remodelling in this population were baseline LVEF and infarct size assessed by rest perfusion SPECT.

Impact of cell therapy on left ventricular remodelling Recently, the BONAMI trial [7] demonstrated that intracoronary autologous bone marrow cells (BMC) administration to patients with decreased LVEF after MI was associated with a 3 months improvement of myocardial viability. Moreover,
LVEF significantly increased by $4.3 \%(p=0.001)$ in the control group and $3.3 \%(p=0.009)$ in the BMC group between baseline and 3 months, without difference for LVEF at 3 months between the two groups. There was no significant difference as well between patients groups in end-diastolic and end-systolic left ventricular volume. Several metaanalyses established that the improvement of LVEF and ESV in favour of stem cell therapy is significant, but modest (approximately 3-4\% increase for LVEF, and 5-10 $\mathrm{ml} \mathrm{de-}$ crease for ESV), with a high degree of statistical heterogeneity in the comparisons. A dose-response relationship $[6,13,16]$ was found between injected cell volume and LVEF changes, as well as a significant impact of intramuscular cell administration and low baseline LVEF [14, 34]. In the present study, an intracoronary injection of BMC performed after a reperfused acute MI in addition to an optimal state-of-the-art pharmacological therapy did not significantly impact on left ventricular remodelling at 1-year follow-up.

\section{Conclusion}

Patients with large myocardial infarction that resulted in early left ventricular dysfunction are at high risk of ventricular remodelling at 1-year follow-up. Baseline rest perfusion SPECT defect (i.e., infarct size) and left ventricular function, but not LV dyssynchrony were predictive of remodelling in this population. Finally, intracoronary injection of bone marrow cells did not significantly impact on cardiac remodelling.

\section{Compliance with ethical standards}

Funding This work was supported in part by a PHRC (Programme Hospitalier de Recherche Clinique) from the French Department of Health (DGOS), and grants from the Association Française contre les Myopathies and the Fondation de France.

Conflict of interest The authors declare that they have no conflict of interest.

Statement of human rights The ethics review board of Nantes University Hospital approved the protocol, and the study was conducted in accordance with the Declaration of Helsinki. Informed consent was obtained from all individual participants included in the study.

Clinical trial registration information URL: Unique identifier NCT00200707.

\section{References}

1. Bolognese L, Neskovic AN, Parodi G, Cerisano G, Buonamici P, Santoro GM, et al. Left ventricular remodeling after primary coronary angioplasty patterns of left ventricular dilation and long-term prognostic implications. Circulation. 2002;106:2351-7. 
2. Parodi G, Memisha G, Carrabba N, Signorini U, Migliorini A, Cerisano G, et al. Prevalence, predictors, time course, and longterm clinical implications of left ventricular functional recovery after mechanical reperfusion for acute myocardial infarction. Am J Cardiol. 2007;100:1718-22.

3. Nucifora G, Bertini M, Marsan NA, Delgado V, Scholte AJ, Ng ACT, et al. Impact of left ventricular dyssynchrony early on left ventricular function after first acute myocardial infarction. Am J Cardiol. 2010;105:306-11.

4. Zhang Y, Yip GW, Chan AKY, Wang M, Lam WWM, Fung JWH, et al. Left ventricular systolic dyssynchrony is a predictor of cardiac remodeling after myocardial infarction. Am Heart J. 2008;156: 1124-32.

5. Chang S-A, Chang H-J, Choi SI, Chun EJ, Yoon YE, Kim H-K, et al. Usefulness of left ventricular dyssynchrony after acute myocardial infarction, assessed by a tagging magnetic resonance image derived metric, as a determinant of ventricular remodeling. Am J Cardiol. 2009;104:19-23.

6. Zimmet H, Porapakkham P, Porapakkham P, Sata Y, Haas SJ, Itescu $\mathrm{S}$, et al. Short- and long-term outcomes of Intracoronary and endogenously mobilized bone marrow stem cells in the treatment of ST-segment elevation myocardial infarction: a meta-analysis of randomized control trials. Eur J Heart Fail. 2012;14:91-105.

7. Roncalli J, Mouquet F, Piot C, Trochu J-N, Le Corvoisier P, Neuder $\mathrm{Y}$, et al. Intracoronary autologous mononucleated bone marrow cell infusion for acute myocardial infarction: results of the randomized multicenter BONAMI trial. Eur Heart J. 2011;32:1748-57.

8. Manrique A, Bernard M, Hitzel A, Bauer F, Ménard J-F, Sabatier R, et al. Prognostic value of sympathetic innervation and cardiac asynchrony in dilated cardiomyopathy. Eur J Nucl Med Mol Imaging. 2008;35:2074-81.

9. Cerqueira MD, Weissman NJ, Dilsizian V, Jacobs AK, Kaul S, Laskey WK, et al. Standardized myocardial segmentation and nomenclature for tomographic imaging of the heart a statement for healthcare professionals from the cardiac imaging committee of the council on clinical cardiology of the american heart association. Circulation. 2002;105:539-42.

10. Faraggi M, Karila-Cohen D, Brochet E, Lebtahi R, Czitrom D, Feldman LJ, et al. Relationship between resting 201Tl reverse redistribution, microvascular perfusion, and functional recovery in acute myocardial infarction. J Nucl Med. 2000;41:393-9.

11. Lang RM, Bierig M, Devereux RB, Flachskampf FA, Foster E, Pellikka PA, et al. Recommendations for chamber quantification: a report from the american society of echocardiography's guidelines and standards committee and the chamber quantification writing group, developed in conjunction with the european association of echocardiography, a branch of the european society of cardiology. J Am Soc Echocardiogr. 2005;18:1440-63.

12. Sutton MGSJ, Sharpe N. Left ventricular remodeling after myocardial infarction pathophysiology and therapy. Circulation. 2000;101: 2981-8.

13. Lipinski MJ, Biondi-Zoccai GGL, Abbate A, Khianey R, Sheiban I, Bartunek J, et al. Impact of Intracoronary cell therapy on left ventricular function in the setting of acute myocardial infarction: a collaborative systematic review and meta-analysis of controlled clinical trials. J Am Coll Cardiol. 2007;50:1761-7.

14. Brunskill SJ, Hyde CJ, Doree CJ, Watt SM, Martin-Rendon E. Route of delivery and baseline left ventricular ejection fraction, key factors of bone-marrow-derived cell therapy for ischaemic heart disease. Eur J Heart Fail. 2009; 11:887-96.

15. Zhang S, Sun A, Ge J, Yao K, Huang Z, Wang K, et al. Intracoronary autologous bone marrow stem cells transfer for patients with acute myocardial infarction: a meta-analysis of randomised controlled trials. Int J Cardiol. 2009;136:178-85.

16. Martin-Rendon E, Brunskill SJ, Hyde CJ, Stanworth SJ, Mathur A, Watt SM. Autologous bone marrow stem cells to treat acute myocardial infarction: a systematic review. Eur. Heart J. [Internet]. 2008 [cited 2015 Jan 26]; Available from: http:// eurheartj.oxfordjournals.org/content/early/2008/06/03/eurheartj. ehn220

17. Mollema SA, Liem SS, Suffoletto MS, Bleeker GB, van der Hoeven BL, van de Veire NR, et al. Left ventricular dyssynchrony acutely after myocardial infarction predicts left ventricular remodeling. J Am Coll Cardiol. 2007;50:1532-40.

18. Giannuzzi P, Temporelli PL, Bosimini E, Gentile F, Lucci D, Maggioni AP, et al. Heterogeneity of left ventricular remodeling after acute myocardial infarction: results of the gruppo italiano per lo studio della sopravvivenza nell'Infarto miocardico-3 echo substudy. Am Heart J. 2001;141:131-8.

19. White HD, Norris RM, Brown MA, Brandt PW, Whitlock RM, Wild CJ. Left ventricular end-systolic volume as the major determinant of survival after recovery from myocardial infarction. Circulation. 1987;76:44-51.

20. Pfeffer MA, Braunwald E, Moyé LA, Basta L, Brown EJ, Cuddy TE, et al. Effect of captopril on mortality and morbidity in patients with left ventricular dysfunction after myocardial infarction. N Engl J Med. 1992;327:669-77.

21. Doughty RN, Whalley GA, Walsh HA, Gamble GD, LópezSendón J, Sharpe N. Effects of carvedilol on left ventricular remodeling after acute myocardial infarction the CAPRICORN echo substudy. Circulation. 2004;109:201-6.

22. Pitt B, Remme W, Zannad F, Neaton J, Martinez F, Roniker B, et al. Eplerenone, a selective aldosterone blocker, in patients with left ventricular dysfunction after myocardial infarction. N Engl J Med. 2003;348:1309-21.

23. Parodi G, Antoniucci D. Left ventricular remodeling after primary percutaneous coronary intervention. Am Heart J. 2010;160:S11-5.

24. Turan B, Yilmaz F, Karaahmet T, Tigen K, Mutlu B, Basaran Y. Role of left ventricular dyssynchrony in predicting remodeling after ST elevation myocardial infarction. Echocardiography. 2012;29: 165-72.

25. Fahmy Elnoamany M, Mahfouz Badran H, Helmy Abo Elazm T, Shawky Abdelaziz E. Asynchrony of left ventricular systolic performance after the first acute myocardial infarction in patients with narrow QRS complexes: doppler tissue imaging study. J Am Soc Echocardiogr. 2006;19:1449-57.

26. Zhang Y, Chan AKY, Yu C-M, Lam WWM, Yip GWK, Fung W-H, et al. Left ventricular systolic asynchrony after acute myocardial infarction in patients with narrow QRS complexes. Am Heart J. 2005;149:497-503.

27. Nucifora G, Bertini M, Marsan NA, Scholte AJ, Siebelink H-MJ, Holman ER, et al. Temporal evolution of left ventricular dyssynchrony after myocardial infarction: relation with changes in left ventricular systolic function. Eur Heart J Cardiovasc Imaging. 2012;13(12):1041-6.

28. Marsan NA, Henneman MM, Chen J, Ypenburg C, Dibbets P, Ghio $\mathrm{S}$, et al. Left ventricular dyssynchrony assessed by two threedimensional imaging modalities: phase analysis of gated myocardial perfusion SPECT and tri-plane tissue doppler imaging. Eur J Nucl Med Mol Imaging. 2008;35:166-73.

29. Henneman MM, Chen J, Dibbets-Schneider P, Stokkel MP, Bleeker GB, Ypenburg C, et al. Can LV dyssynchrony as assessed with phase analysis on gated myocardial perfusion SPECT predict response to CRT? J Nucl Med. 2007;48:1104-11.

30. Miller TD, Christian TF, Hopfenspirger MR, Hodge DO, Gersh BJ, Gibbons RJ. Infarct size after acute myocardial infarction measured by quantitative tomographic $99 \mathrm{mTc}$ sestamibi imaging predicts subsequent mortality. Circulation. 1995;92:334 41.

31. Bolognese L, Cerisano G, Buonamici P, Santini A, Santoro GM, Antoniucci D, et al. Influence of infarct-zone viability on left ventricular remodeling after acute myocardial infarction. Circulation. 1997;96:3353-9. 
32. Lipiecki J, Cachin F, Durel N, de Tauriac O, Ponsonnaille J, Maublant J. Influence of infarct-zone viability detected by rest Tc-99 m sestamibi gated SPECT on left ventricular remodeling after acute myocardial infarction treated by percutaneous transluminal coronary angioplasty in the acute phase. J Nucl Cardiol. 2004;11: 673-81.

33. Berti V, Sciagrà R, Acampa W, Ricci F, Cerisano G, Gallicchio R, et al. Relationship between infarct size and severity measured by gated SPECT and long-term left ventricular remodelling after acute myocardial infarction. Eur J Nucl Med Mol Imaging. 2011;38: 1124-31.

34. Schächinger V, Erbs S, Elsässer A, Haberbosch W, Hambrecht R, Hölschermann $\mathrm{H}$, et al. Intracoronary bone marrow-derived progenitor cells in acute myocardial infarction. N Engl J Med. 2006;355:1210-21. 